\title{
Targeted fascicular biopsy in a patient with prostate cancer
}

\author{
Jonathan J. Stone, MD, Megan C. Kaszuba, MD, and Robert J. Spinner, MD
}

Department of Neurologic Surgery, Mayo Clinic, Rochester, Minnesota

Patients who present with a history of cancer and the new onset of lumbosacral or peripheral neuropathy should be evaluated for the potential of metastasis. Targeted fascicular biopsy can be useful to diagnose atypical lesions within peripheral nerves in patients with major or progressive neurological deficits. In this video, the authors demonstrate the technique of targeted fascicular biopsy of the sciatic nerve in a 63-year-old man with a history of prostate cancer.

The video can be found here: https://youtu.be/PTOX9XxNBDU.

KEYWORDS peripheral nerve; prostate cancer; targeted fascicular biopsy; perineural spread; video 